\title{
Better Frame Rates or Better Visuals? An Early Report of Player Practice in Esports
}

\author{
Arjun Madhusudan \\ North Carolina State University \\ Raleigh, North Carolina, USA \\ amadhus2@ncsu.edu
}

\author{
Benjamin Watson \\ North Carolina State University \\ Raleigh, North Carolina, USA \\ bwatson@ncsu.edu
}

\begin{abstract}
Esports athletes often reduce visual quality to improve latency and frame rate, and increase their in-game performance. While limited research has examined the effects of this visuo-spatial tradeoff on performance, we could find no work studying how widespread this practice is, and how it is done. This paper describes an initial examination of these questions focused on Dota 2, one of the leading esports games. First, we study how athletes manage the visuotemporal tradeoff in practice by gathering the game configuration data of Dota 2 players in a small survey. We learn that players do limit visual detail, particularly by turning off VSYNC, which removes rendering/display synchronization delay but permits visual "tearing". Second, we study the intent of those same players with a few subjective questions, finding that though there were exceptions, player intent and practice aligned well. While our sampling of Dota 2 players was likely not representative, our survey did reveal suggestive trends that lay the groundwork for future, more rigorous and larger surveys.
\end{abstract}

\section{CCS CONCEPTS}

-Information systems $\rightarrow$ Massively multiplayer online games - Software and its engineering $\rightarrow$ Interactive games; $•$ Humancentered computing $\rightarrow$ Empirical studies in $\mathrm{HCI}$.

\section{KEYWORDS}

esports, frame rate, texture, shadows, spatial, temporal, performance, Dota 2, visual detail, temporal detail, gaming, video games, MOBA, latency, level of detail

\section{ACM Reference Format:}

Arjun Madhusudan and Benjamin Watson. 2018. Better Frame Rates or Better Visuals? An Early Report of Player Practice in Esports. In Proceedings of ACM Conference (Conference'17). ACM, New York, NY, USA, 5 pages. https://doi.org/10.1145/1122445.1122456

\section{INTRODUCTION}

With high-speed internet, online gaming has become increasingly common, giving rise to a professional esports industry that rivals traditional sports. Like those traditional sports, esports has begun

Permission to make digital or hard copies of all or part of this work for personal or classroom use is granted without fee provided that copies are not made or distributed for profit or commercial advantage and that copies bear this notice and the full citation on the first page. Copyrights for components of this work owned by others than ACM must be honored. Abstracting with credit is permitted. To copy otherwise, or republish, to post on servers or to redistribute to lists, requires prior specific permission and/or a fee. Request permissions from permissions@acm.org.

Conference'17, fuly 2017, Washington, DC, USA

(c) 2018 Association for Computing Machinery.

ACM ISBN 978-x-xxxx-xxxx-x/YY/MM...\$15.00

https://doi.org/10.1145/1122445.1122456 driving down the price of its technologies, making graphics, display and input equipment available at low prices for casual gamers.

As in most sports, elite esports athletes seek an edge, while casual athletes are just trying to compete. For both, this can mean improved temporal detail, in the form of frame rate and latency; or better visual detail, such as additional rendering or animation. Prevailing opinion in the esports community prefers temporal over visual detail. For example, many advocate turning off VSYNC, which increases frame rate and reduces latency by removing synchronization delay between the graphics processing unit (GPU) and the display (e.g. [7]). The tradeoff introduced with this improvement is tearing, as two or more frames - each showing a different moment in time - appear on the display.

There has been limited study of how temporal and visual detail combine to affect performance in games, but to our knowledge no extant research exists on how players actually make this tradeoff in practice. We believe that the burgeoning number of esports athletes wrestling with this same question provides a unique research opportunity. In this paper, we describe a pilot study of how esports athletes manage the visuo-temporal tradeoff in their own gameplay.

\section{VISUAL AND TEMPORAL DETAIL}

In computer games, configurable visual detail typically includes features that players can see, but have limited effect on gameplay. Instead, they are primarily a way of making the game more pleasing, emphasize the fantasy element of the game-world. Visual detail typically includes options like higher-resolution texture, ambient occlusion, more accurate lighting, special animation, and even UI elements such as an animated/live heads-up display (HUD).

Options for directly configuring temporal detail are more limited, and can include VSYNC (de)activation and frame rate caps. Instead, because visual and temporal detail are almost always in tradeoff, players often configure temporal detail indirectly by manipulating visual detail. For example, a player might lower texture detail to increase frame rate and reduce latency. Both the research and the esports communities believe that temporal detail plays a major role in gaming performance.

Esports players can configure settings and the visuo-spatial tradeoff as they like, but can become quite confused while doing so: each of the dozens of relevant settings is hard to understand and difficult to find among hundreds of other irrelevant settings.

\section{RELATED WORK}

Improved temporal detail has long been found to improve human performance in general [2]. In games, reducing latency and increasing refresh rates often translates into winning. For example, 240 
$\mathrm{Hz}$ monitors and adaptive sync have proven to be useful to players $[5,11]$, even when they could not tell when they were using them.

Research on how visual detail affects performance is limited. Claypool et al. [3, 4] are among the few who examined a possible relationship, and could not confirm it. They did however find a relationship to player experience, and indeed visual elements play an important role in the gaming economy. Free to play games like Dota 2 thrive on monetization of virtual goods. These include nonfunctional animations, effects, and in-game cosmetics such as player skins and avatars [6]. Their quality is highly influenced by detail settings, giving players an incentive to use higher visual detail.

Even less research addresses the combined effect of temporal and visual detail on gaming performance, with Claypool et al. [3, 4] finding further that rendering resolution did not interact with frame rate's effects. This is a stark contrast with research in streaming video, in which resolution had a larger effect than frame rate.

While most research on the visuo-temporal tradeoff in gaming has examined its effect on player performance, this paper focuses on how players choose to make this tradeoff in their own gaming.

\section{DATA COLLECTION}

We chose to study the visuo-temporal detail tradeoff in the context of Dota 2, one of the most popular esports games. Dota pits networked opponents against one another in a 3D environment, with the goal of taking the opponent's base. The quick pace of the game provides an advantage to the first mover, and rewards players with quick reflexes and faster computer systems. The game has over one million active players, with its largest annual tournament “The International" awarding a prize pool of $\$ 40,000,000$ in 2020. In addition, it adopts a multiplayer online battle arena style of play, which is quite common among the most significant esports games.

To collect information regarding the in-game configurations of Dota 2 players, we recruited in online forums such as Reddit. We asked respondents to submit their Dota 2 configuration files, each of which describes over 450 in-game settings. We requested that players obtain these files from the same system they used to play Dota 2. We also asked respondents to answer a three-question subjective survey:

- Have you played Dota 2 competitively (on any scale)?

- Which setting would you reduce to increase computer performance (reduce lag/system crashes etc)?

- Which setting would you reduce to increase your own performance (details that might distract you or make it harder to see while playing etc)?

To the first question, respondents could reply "no" or "yes," followed by a short description of the tournament. To answer the second and third questions, respondents ordered four options: "shadows," "textures," "screen size," or "FPS" (frames per second).

\subsection{Participants}

Our study was open to any gamer who used Windows to play Dota 2 , and who actively played Dota 2 on any level, whether casual or professional. No personal information was collected, and no compensation was provided. We received 43 responses, with one respondent submitting an invalid configuration file.
We do not claim that our sample of 43 players is representative of the Dota 2 player base, which numbers in the millions [9], and is unusually international [1]. Nevertheless, our preliminary survey does suggest trends and raise questions that might be followed up in more rigorous future surveys.

\section{RESULTS}

We begin by studying the configuration files we collected, and then examine responses to our three-question subjective survey.

\subsection{Configuration results}

From the over 450 settings in each configuration file, we focused on 15 that affected either visual or temporal game detail, and which players could easily change through the in-game configuration screen (see Table 1).

Table 1: The 15 configuration settings that met our relevance criteria. Columns from left to right show the setting identifier, a sparkline of that setting's participant data, and the proportion of that setting's data that limited visual detail.

Setting Name
mat_vsync
Cl_particle_fallback_base
r_texture_stream_mip_bias
Cl_globallight_shadow_mode
dota_cheap_water
r_ssao
dota_ambient_creatures
r_deferred_specular_bloom
dota_ambient_cloth
r_deferred_specular
r_dota_allow_wind_on_trees
r_grass_quality
r_deferred_additive_pass
r_deferred_simple_light
mat_viewportscale

Definitions of some key settings include:

- useadvanced: Switches between simple and complex setting controls. 0 uses presets provided by the game to manage settings as a group, while 1 allows the player to customize each setting individually.

- mat_vsync: Indicates whether the player has VSYNC on or off. When VSYNC is on, GPU frames and display refreshes are synchronized, reducing frame rate but limiting tearing. When it is off, frame rate increases but tearing is visible.

- r_texture_stream_mip_bias: Changes texture resolution in the mipmap. 0 uses coarse textures, 1 medium, and 2 fine. We considered values 0 and 1 as reduced visual detail.

- mat_viewportscale: Scaling factor for the viewport. If less than 1, it limits visual detail by rendering fewer pixels.

- cl_particle_fallback_base: Controls level of detail in special effects such as glow, particles, and smoke, with 0 being low, 2 being medium and 4 being high. We considered values 0 and 2 as limiting visual detail, while 4 did not.

While we could not locate one definitive resource that clearly defines all Dota 2 settings, most can be found in online Dota 2 
forums $[8,10]$. With a few exceptions (texture_stream_mip_bias, mat_viewportscale, cl_globallight_shadow_mode, and particle_fallback_base), all settings had two possible values.

Table 1 contains sparklines. While they are traditionally used to show variation over time, we use them to visualize similarities between participant settings. Their horizontal axis indicates the different players, whereas their vertical axis represents setting value (mostly, on or off).

The only settings with trends exceeding $60 \%$ limited visuals: vsync, texture_stream_mip_bias and particle_fallback_base. In particular, vsync was turned off by over $80 \%$ of our participants, improving temporal detail (i.e. frame rates, latency) at the cost of visual detail (tearing).

Table 1's similar sparklines reveal that most participants configured most settings similarly. Many settings are configured automatically by Dota 2 or graphics drivers. To get a better sense of which settings were then altered directly by our participants, we considered only those players who used advanced mode (useadvanced) to customize individual Dota 2 settings. useadvanced is never enabled on installation, meaning a participant must act to enable it, making it more likely that they will determine other settings manually.

Table 2: The same 15 configuration settings as in Table 1, but only for participants who are likely more familiar with them, and chose to use advanced settings.

\begin{tabular}{l} 
Setting Name \\
\hline mat_vsync \\
r_texture_stream_mip_bias \\
cl_particle_fallback_base \\
Cl_globallight_shadow_mode \\
dota_cheap_water \\
r_ssao \\
dota_ambient_creatures \\
r_dota_allow_wind_on_trees \\
dota_ambient_cloth \\
r_grass_quality \\
r_deferred_specular \\
r_deferred_specular_bloom \\
r_deferred_additive_pass \\
r_deferred_simple_light \\
mat_viewportscale
\end{tabular}

Table 2 shows how the same 15 settings were made by participants that enabled useadvanced. The settings show strengthening trends: now both vsync and texture_stream_mip_bias limit visuals for more than $80 \%$ of players, and particle_fallback_base nearly does the same. In addition, new trends limiting temporal detail emerge: viewportscale, deferred_additive_pass and simple_light all limit temporal detail (and improve visual detail) for more than $60 \%$ of players. Participants' settings were also slightly less consistent, with the cheap_water setting now more different from following settings than among the full 42 participants.

\subsection{Survey results}

Our participants represent competitive and casual gamers in roughly equal proportion. Of the 43 players who responded, 21 players (48.8\%) played the game competitively, meaning they took part in tournaments on various levels such as school, nation or online competitive platforms such as FaceIt.

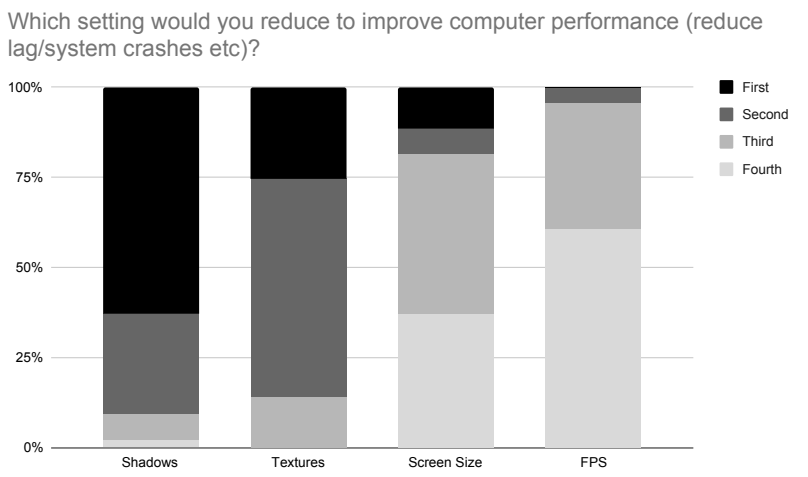

Figure 1: Participants' orderings of methods for improving computer performance.

Participants' answers to both the second ("improve computer performance") and third ("improve your own performance") questions were quite similar. Figure 1 shows participants' answers to the second question. 27 players $(62.7 \%)$ said shadows would be the first thing they would reduce to improve their system's performance, while 26 players $(60.5 \%)$ also agree that limiting FPS would be last on their list. 35 players (81.4\%) would not place reducing screen size first or second on their list.

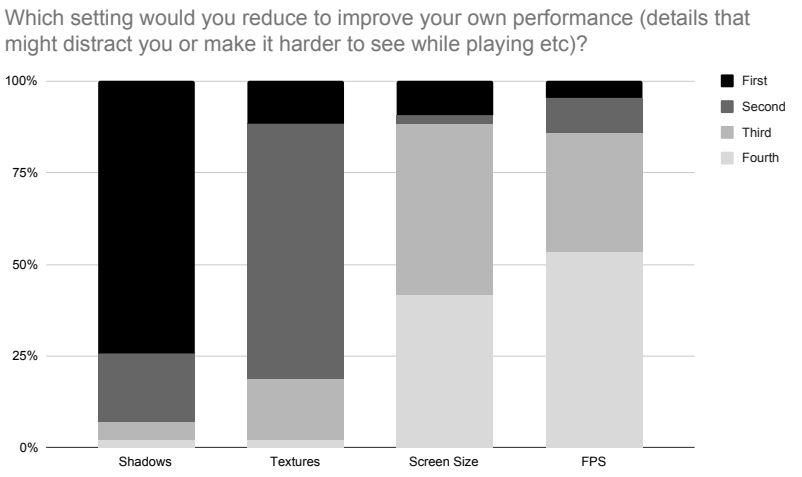

Figure 2: Participants' orderings of methods for improving their gaming performance.

Figure 2 shows participants' answers to the third question. 32 players $(74.4 \%)$ said they would first reduce shadows to improve their own performance, while 23 players (53.3\%) agreed that limiting FPS would be their last option. 38 players $(88.4 \%)$ did not place reducing screen size first or second on their list.

\section{DISCUSSION}

We begin our discussion of these results by discussing the many reasons for caution in interpreting them. We then move on to possible explanations and implications. 


\subsection{Limitations}

There are many features in this work that limit its generality, and suggest possible future work. We discuss a few of these here.

First, our 43 respondents are likely not a representative sample of the Dota 2 player population, which numbers in the millions. Despite this large population, careful application of survey methodology should allow future researchers to obtain a representative sample with responses numbering in the low thousands. As groundwork for such an effort, we need a good sense of Dota 2 demographics across (perhaps) nationality, game familiarity, age and gender an "esports census".

Next, we cannot be certain whether settings result from conscious player choice, or are automated system defaults. Future studies might ask respondents to identify those settings they made themselves. Information about respondent hardware configurations (e.g. CPU, GPU, display) might also set these settings and choices in an informative context.

In addition, even when players make conscious choices, we cannot be certain that they understood their implications. As this paper begins to make clear, the range of settings and possible interactions they might have with one another, hardware, and gameplay can be overwhelming. For example, a player might choose a setting because they believe it limits a visual effect and improves frame rate, but they are quite mistaken. Future work might query player understanding of Dota 2 settings.

Finally, Dota 2 is just one of several dominant esports games, and one of hundreds of recently significant titles in the broader gaming industry. These games can vary significantly in genre (e.g. battle area vs. first-person shooter), task and more. The trends we find here may not be found in other games.

\subsection{Possible explanations}

In our three-question survey, the similarity between replies to the "improve computer performance" and "improve your own performance" questions suggests that participants associated gaming performance with computer performance, not with visual quality: the faster their system ran, the better they would play. We can see these survey results reflected in respondents configuration settings as well: textures and shadows are usually limited. However, in both the survey and configuration settings, rendered screen size (viewportscale) was usually not reduced, despite the improvements in temporal detail that would result. It may be that this particular type of visual detail - the number of pixels rendered, which has a much more global visual impact than other types, such as texture or shadows - is especially valuable to players.

The strongest trend in our configuration results is the widespread deactivation of VSYNC, which improves temporal detail as measured by frame rates and latencies, but harms visual detail by introducing a tear into displayed imagery between frames representing different moments in time. Players seem quite willing to sacrifice temporal coherence for more up-to-date information. To our knowledge, these are the first (admittedly preliminary) data describing this preference.

The remaining strong trends in our configuration settings data indicate that players regularly limit visual effects such as texture resolution and animated particle complexity to improve temporal detail. However, weaker trends among better informed and more proactive players (using advanced settings) show that players sometimes improve visual effects (e.g. by maintaining screen size or using an additive rendering pass). As we speculated with our screen size discussion above, it may be that certain types of visual detail are particularly important. Alternatively, they may have only a minor impact on temporal detail and/or player performance.

In both our broader and advanced configuration data, we observed a great deal of within-player consistency, that is, players consistently $\mathrm{did} / \mathrm{did}$ not limit visual detail. This might reflect individual player goals, or alternatively, consistent defaults made by the player's system.

\subsection{Implications}

We consider the implications of our pilot esports configuration survey for for players, designers and researchers.

Players should prioritize improvements in temporal detail when configuring their games. Prior research on temporal detail's effects on gaming performance shows that better frame rates and latencies improve gaming performance, and indeed in our survey the configurations of most players prioritize improvements in frame rates. Interestingly however, not all reductions in visual detail are justified; players should carefully evaluate each setting's effect on gameplay.

Given the importance of temporal detail for game performance, designers may wish to offer additional settings with good "tempo$\mathrm{ral} / \mathrm{visual}$ ratios", that is, that maximize the temporal benefits and minimize the visual penalties for gaming performance. Additionally, the obtuse and tangled collection of settings can easily overwhelm both players and researchers: please simplify and explain!

In addition to scaling and generalizing this work, researchers might investigate why some visual tradeoffs were made (e.g. $r_{-}$texture_stream_mip_bias) and others were not (e.g. mat_vsync). Could it be related to gaming experience, as opposed to performance? And finally, the complex web of detail settings is a constant source of frustration and confusion for users. Solving this problem could be important not only for esports but also for potential applications of its technology in video conferencing, remote operation and telemedicine.

\section{CONCLUSION AND FUTURE WORK}

This paper presents a unique but preliminary investigation of the visuo-temporal tradeoffs esports athletes make in configuring their own Dota 2 games. We find trends indicating that with a few exceptions, players prioritize temporal detail (better frame rates and latencies) over visual detail (better visual effects). In particular, most of the athletes we studied turned VSYNC off to improve temporal detail, despite the visual tearing this introduces.

Future work should seek to confirm these trends with a more representative survey sample, and generalize them by studying other games. There is a great deal of confusion among both researchers and athletes in understanding the impact of the many configuration settings available in most games, and any efforts at improving clarity would be most welcome. Finally, we hope that any lessons learned in manipulating detail to improve esports performance would be applied to improve expert performance in general. 


\section{REFERENCES}

[1] Carto. 2021. Number of Dota 2 players per country. hhttps://kassgrain.carto.com/ viz/c5380a68-2bc2-11e6-b452-0ecfd53eb7d3/public_map

[2] Jessie YC Chen and Jennifer E Thropp. 2007. Review of low frame rate effects on human performance. IEEE Transactions on Systems, Man, and Cybernetics-Part A Systems and Humans 37, 6 (2007), 1063-1076.

[3] Mark Claypool and Kajal Claypool. 2009. Perspectives, frame rates and resolutions: it's all in the game. In Proceedings of the 4th International Conference on Foundations of Digital Games. 42-49.

[4] Mark Claypool, Kajal Claypool, and Feissal Damaa. 2006. The Effects of Frame Rate and Resolution on Users Playing First Person Shooter Games. Proceedings of SPIE - The International Society for Optical Engineering 6071 (01 2006). https: //doi.org/10.1117/12.648609

[5] Joohwan Kim, Josef Spjut, Morgan McGuire, Alexander Majercik, Ben Boudaoud, Rachel Albert, and David Luebke. 2019. Esports Arms Race: Latency and Refresh Rate for Competitive Gaming Tasks. fournal of Vision 19, 10 (2019), 218c.
[6] Ilya Musabirov, Denis Bulygin, Paul Okopny, and Alexander Sirotkin. 2017. Deconstructing Cosmetic Virtual Goods Experiences in Dota 2. In Proceedings of the 2017 CHI Conference on Human Factors in Computing Systems (Denver, Colorado, USA) (CHI '17). Association for Computing Machinery, New York, NY, USA, 2054-2058. https://doi.org/10.1145/3025453.3025893

[7] pcgamehaven.com. 2020. What is VSync? Should you disable it, or enable it? https://pcgamehaven.com/what-is-vsync-should-you-disable-it-or-enable-it/

[8] Reddit.com. 2013. Is there a way to increase graphical quality through the config? https://www.reddit.com/r/DotA2/comments/15tyoo/is_there_a_way_to_ increase graphical quality/c7puu $5 \mathrm{~s}$

[9] Steamcharts.com. 2021. Dota 2 - Steam Charts. https://steamcharts.com/app/570

[10] Steamcommunity.com. 2016. Optimization / Tweak for DotA 2 Gaming. https: //steamcommunity.com/sharedfiles/filedetails/?id=339319323

[11] Benjamin Watson, Rachit Shrivastava, and Ajinkya Gavane. 2019. The Effects of Adaptive Synchronization on Performance and Experience in Gameplay. Proc. ACM Comput. Graph. Interact. Tech. 2, 1, Article 5 (June 2019), 13 pages. https: //doi.org/10.1145/3320286 